\title{
The portable device improves the detection of atrial fibrillation after ablation
}

\author{
Songqun Huang ${ }^{1}$, Teng Zhao ${ }^{1}$, Chao Liu ${ }^{1}$, Aihong Qin ${ }^{1}$, Shaohua Dong ${ }^{1}$, Binhang Yuan ${ }^{2}$, \\ Wenhui Xing ${ }^{3}$, Zhifu Guo ${ }^{1}$, xin-miao huang ${ }^{4}$, Yong-Mei Cha ${ }^{5}$, and Jiang Cao ${ }^{1}$ \\ ${ }^{1}$ Changhai Hospital \\ ${ }^{2}$ William Marsh Rice University \\ ${ }^{3}$ Shanghai Yueguang Medical Technologies Ltd \\ ${ }^{4}$ Changhai Hospital, Second Military Medical University \\ ${ }^{5}$ Mayo Clinic
}

August 4, 2020

\begin{abstract}
Background: Asymptomatic recurrences of atrial fibrillation (AF) are common after ablation of AF. Objective: We aimed to analyze the performance of the mobile ECG device using artificial intelligence (AI) algorithm in detection of AF after ablation. Method: A randomized controlled trial of AF screening using a handheld single-lead ECG monitor (BigThumbß) or a traditional follow-up strategy was conducted in patients with non-valvular AF after catheter ablation. Consecutive patients were randomized to either BigThumb Group (BT Group) or Traditional Follow-up Group (TF Group). Monitoring data was collected and analyzed. The ECGs collected by BigThumb were compared using the automated AF detection algorithm, AI algorithm and cardiologists' manual review. Subsequent changes in adherence on oral anticoagulation of patients were also recorded. Result: We studied 218 patients (109 in BT Group, 109 in TF Group). After a follow-up of 345.4 \pm 60.2 days, AF-free survival rate was $64.2 \%$ in BT Group and $78.9 \%$ in TF Group ( $\mathrm{P}=0.0163)$, with more adherence on oral anticoagulation in BT Group ( $\mathrm{P}=0.0052)$. The participants in the BT Group recorded 26133 ECGs during the follow-up, among which 3299 (12.6\%) were diagnosed as AF by cardiologists' manual review. The sensitivity and specificity of the AI algorithm were $94.4 \%$ and 98.5\% respectively, which are significantly higher than the automated AF detection algorithm (90.7\% and 96.2\%). Conclusion: We found that follow-up after AF ablation using BigThumb leads to a more frequent detection of AF recurrence and more adherence on oral anticoagulation. Artificial intelligence algorithm improves the accuracy of ECG diagnosis.
\end{abstract}

\section{Introduction}

Atrial fibrillation (AF) is one of the most common arrhythmias, affecting an estimated 33.5 million patients worldwide $^{1}$. It is a serious public health problem because of its increasing incidence and prevalence in the aging population and its association with elevated risks of cardiovascular events and death ${ }^{2}$. Catheter ablation is increasingly common in AF treatment because of reduction of symptoms. However, asymptomatic recurrence of AF after ablation is substantial and a frequent monitoring strategy is warranted for early detection of $\mathrm{AF}$ episode and subsequent change of medical treatment ${ }^{3}$.

Asymptomatic AF is difficult to diagnose based on a short electrocardiography (ECG) recording, especially when the episode is paroxysmal. It has been demonstrated that traditional monitoring methods, including ECG and Holter monitoring, may overestimate the effectiveness of catheter ablation ${ }^{4}$. The portable devices used to monitor AF and artificial intelligence (AI) algorithm applied has the potential to improve accuracy in rhythm monitoring and diagnosis. 
We therefore undertook a randomized controlled trial with the portable device versus traditional followup strategy in a post-ablation population to evaluate the feasibility and veracity of the self-applied ECG monitoring device in detection of atrial fibrillation.

\section{Methods}

The study was designed as a randomized controlled single-center study.

\subsection{Patients}

Patients aged $>18$ years with symptomatic AF refractory to at least one class I or class III antiarrhythmic drug, and referred for a first catheter ablation procedure at Changhai Hospital between March 2019 and October 2019 were enrolled. All patients provided written informed consent. Consecutive 218 patients were randomized to either BigThumb Group (BT Group) or Traditional Follow-up Group (TF Group) by means of random number table. The baseline clinical features were noted for each patient and compared for different follow-up strategies. The study was conducted in accordance with the Declaration of Helsinki. The study was approved by the institutional review board of Shanghai Changhai Hospital, Second Military Medical University.

\subsection{Ablation procedures}

For all cases, standard femoral venous access was achieved using Seldinger technique with a 6-French and a 7-French sheath in the left femoral vein and two 8.5-French Swarz sheaths in the right femoral vein. Coronary sinus catheter and right ventricle catheter were advanced through left femoral vein. Transseptal access was achieved with fluoroscopic guidance. A Tacticath Quartz catheter (St Jude Medical, St. Paul, MN, USA) was used for radiofrequency catheter ablation in each procedure. The ablation strategy for each case was left to the discretion of the primary operator.

\subsection{The BigThumb heart monitor}

The technologies used to monitor AF are advancing at a rapid pace. Among them, the BigThumb (Shanghai Yueguang Medical Technologies Inc, Shanghai, China) heart monitor is a National Medical Products Administration (NMPA) approved smartphone handheld tool that permits easy and rapid collection of a "one-lead" ECG (bECG) with two thumbs of users on the device (Figure 1). In parallel, the bECG will also be sent to doctors automatically. Users can store and review bECGs on their cellphones. Artificial intelligence (AI) algorithm was applied in new version of BigThumb (Supplemental information 1). It may improve the accuracy of automatic diagnosis. The feasibility of the BigThumb applied in follow-up of patients after ablation was characterized.

\subsection{Follow-Up Strategies}

Patients in BT Group were provided with and trained to use a BigThumb ECG monitor, and were instructed to take at least three bECGs every day and additional bECGs if symptomatic. The patients should mark symptoms when bECGs were collected. AF was detected by both an automated AF detection algorithm and an AI algorithm. Two cardiologists will confirm the diagnosis separately. While AF recurrence of patients in TF Group were monitored with Holters 3, 6 and 12 months after ablation and ECGs if there are symptoms. Monitoring data and patient management strategies were collected and analyzed. A blanking period was defined as three months after ablation. Oral anticoagulation was recommended after the "blanking period" when the $\mathrm{CHA}_{2} \mathrm{DS}_{2}$-VASc score $>1$. Subsequent adherence on oral anticoagulation of patients were also recorded.

\subsection{Statistical Analysis}

Continuous variables are expressed as mean \pm standard deviation, and categorical variables are expressed as a number and percentage. Continuous variables were compared using the two-tailed Student's t-test, and categorical variables were compared using the $\chi^{2}$ test or Fischer's exact test. In order to investigate the influence of follow-up strategies on AF recurrence detection, Kaplan-Meier survival curve and log rank 
analysis were performed. For Cox regression, univariable regression was performed first. Variables with a $\mathrm{P}$-value of $<0.2$ were included in the multivariable model. A level of significance was set at $<0.05$ for all reported P-values, and confidence intervals were calculated at the $95 \%$ level. All statistics were calculated using SPSS (Version 24, IBM, Armonk, NY, USA).

\section{Results}

\subsection{Baseline Characteristics}

There were 109 patients in each group. No statistical difference was detected in demographic data between patients in the two groups (Table 1).

\subsection{Primary Outcome}

After follow-up of $344.0 \pm 60.5$ days in BT Group, 70/109 patients $(64.2 \%)$ were free from AF recurrence (70.8\% in paroxysmal $\mathrm{AF}$ and $54.5 \%$ in persistent $\mathrm{AF}$ ). While in $\mathrm{TF}$ Group, the $\mathrm{AF}$ free rate was $86 / 109$ patients $(78.9 \%, \mathrm{P}=0.0163<0.05)$ after follow-up of $346.8 \pm 59.9$ days $(88.2 \%$ in paroxysmal $\mathrm{AF}$ and $57.6 \%$ in persistent $\mathrm{AF}$ ). The BigThumb detected more AF recurrence in paroxysmal AF after ablation $(\mathrm{P}=0.0099<0.05)$ but not in persistent $\mathrm{AF}(\mathrm{P}=0.7910>0.05)$.

Adherence on anticoagulation in patients with $\mathrm{CHA}_{2} \mathrm{DS}_{2}$-VASc score $>1$ was significantly higher in BT Group $(25 / 49,51.0 \%)$ than TF Group $(16 / 63,25.4 \%, \mathrm{P}=0.0052<0.05)$. There were $11 / 109(10.1 \%)$ and $2 / 109(1.8 \%)$ patients receiving a second ablation in BT Group and TF Group respectively $(\mathrm{P}=0.0101<0.05)$.

The Kaplan-Meier curves for recurrence stratified by follow-up strategies at time of enrollment are shown in Figure 2. Log rank tests showed a significantly earlier documentation of AF recurrence in BT Group than TF Group $(\mathrm{P}<0.05)$. In a multivariate Cox model, the likelihood of recurrence detection was greater in patients in BT Group with larger left atrium (Table 2).

\subsection{Feasibility of BigThumb in follow-up after ablation}

A total of 26133 bECGs were recorded, among which 3299 (12.6\%) were confirmed as AF by cardiologists' manual review, 3860 (14.8\%) were diagnosed as AF by the automated AF detection algorithm and 3457 $(13.2 \%)$ by the AI algorithm. The sensitivity of the AI algorithm was significantly higher than the automated AF detection algorithm $[(94.4 \%, 95 \%$ CI, 93.5\% - 95.1\%) VS (90.7\%, 95\% CI, 89.7\% - 91.7\%)]. The specificity was also higher in the AI algorithm [(98.5\%, 95\% CI, 98.3\% - 98.6\%) VS (96.2\%, 95\% CI, 95.9\% - 96.4\%)]. There were $2514 / 3299(76.2 \%)$ AF bECGs marked with symptoms by participants.

\subsection{Compliance of BigThumb Monitoring}

The monitoring frequency was $0.53+-0.02 /$ day in all the participants. They were used more frequently in the first three-month follow-up than after $(1.19+-0.03$ /day VS $0.36+-0.02 /$ day, $\mathrm{P}<0.05)$ as shown in Figure 3 . The monitoring was most frequently collected in the daytime than the nighttime. Hence, the episodes of $\mathrm{AF}$ were more frequently detected during daytime (Figure 4).

\section{Discussion}

To our knowledge, this is the first study to demonstrate the feasibility and veracity of the BigThumb monitoring device. The primary finding of this study is that follow-up after AF ablation using BigThumb leads to a more frequent detection of AF recurrence and more adherence on oral anticoagulation. Artificial intelligence algorithm improves the accuracy of ECG diagnosis.

It was reassuring to see abundant devices and applications available in post-ablation follow-up. ECG and Holter monitoring are the most frequently applied techniques to detect AF recurrence in previous studies. However, low-frequency monitoring is not sufficient for follow-up ${ }^{5-6}$. Pulse wave monitor devices are also used in AF detection with unsatisfied accuracy ${ }^{7-8}$.

Accumulating evidence and meta-analyses have demonstrated that insertable cardiac monitor (ICM) and cardiac implantable electronic device (CIED) detect a high rate of AF typically missed during routine clinical 
care in patients after ablation ${ }^{9-11}$. Early detection of AF is important to define or change proper medical treatment ${ }^{12}$. However, implantation of the devices is invasive and expensive which prevent them from the popularity in follow-up.

AliveCor is a monitor attached to a WiFi-enabled iPod to obtain ECGs in ambulatory patients ${ }^{13}$. It brings contextualized medical-grade ECG technology to the consumer and will inevitably improve AF detection compared with current practice ${ }^{14}$. Halcox et al conducted a randomized controlled trial of AF screening using AliveCor to obtain ECGs ${ }^{15}$. Among the 60440 ECG tracings, only 1\% (600 ECGs) were categorized as $\mathrm{AF}$ by the automated AliveCor algorithm. Of these, only 5\% (30 tracings) were confirmed to be AF. The low positive predictive value of the AliveCor for the detection of $\mathrm{AF}$ was an unexpected observation and made the current generation of AliveCor devices as a screening tool difficult to justify ${ }^{16}$. Similarly, the BigThumb, used for smartphone-based ECG collection, paralleled the AliveCore in structure design, but the sensitivity and specificity of the AI algorithm were $94.4 \%$ and $98.5 \%$. It has the potential to reduce the manual review. The use of mobile ECG self-recording devices allows for earlier detection of AF recurrence and may empower patients to engage in shared health decision-making.

There is emerging evidence that AF recurrence after ablation may be asymptomatic. Studies have shown that asymptomatic AF may be increasingly common after ablation and potentially pose a greater thromboembolic risk than symptomatic $\mathrm{AF}^{17-19}$. In the present study, we found that 785/3299 (23.8\%) of $\mathrm{AF}$ detected after ablation were asymptomatic, that may explain the poor compliance with anticoagulation after catheter ablation even if patients receive recommendations of anticoagulation from doctors. The BigThumb may help to record the episode of asymptomatic AF according to random monitoring which may lead to subsequent adherence on anticoagulation.

The BigThumb is an effective and affordable public ECG monitoring device. This is critical for successful incorporation of consumer-generated biometrics into clinical practice. However, it has limitations. ECGs tracings in this clinical trial provide an insight into the real-world limitations of the technology. Because of random and haphazard collection of bECGs, the accuracy of AF detection with the BigThumb highly depends on the compliance of participants. As we found in this study, the BigThumb was used frequently in the first 3-month follow-up than after 3-month. Patients should be reminded to persistently utilize the device. We also found in this study that the frequency of monitoring in the night was significantly lower than the daytime as expected. Some asymptomatic episodes in the night may be missed. Moreover, despite the best intentions of a health-conscious and motivated individual, the potential for heightened concern with abnormal or equivocal readings remains.

In conclusion, we found that follow-up after AF ablation using BigThumb with artificial intelligence algorithm is more effective than traditional strategies. It is conceivable that in the near future, most patients after ablation will be continuously and indefinitely monitored.

\section{Limitations}

This study has limitations. First, this study is a single center clinical trial with limited follow-up period and comparatively small simple size that may introduce patient selection bias. Second, the clinical setting was specific to China, so our results should be understood in that context. Finally, because there were few episodes of thromboembolism or bleeding, our study does not have sufficient power to detect a difference with the low rate of complications observed according to different clinical decisions after ablation guided by the BigThumb. High-quality research is needed to understand the efficacy of coagulation strategies guided by the BigThumb.

Funding The national natural science foundation of China (81970278)

Acknowledgement We thank Professor Jiang Cao and Yongmei Cha for their valuable discussion and comments. This study was financially supported by the National Natural Science Foundation of China (81970278).

Conflict of interest The authors declare that they have no conflict of interest. 


\section{Reference}

1. Chugh SS, Havmoeller R, Narayanan K, Singh D, Rienstra M, Benjamin EJ, Gillum RF, Kim YH, McAnulty JH, Jr., Zheng ZJ, Forouzanfar MH, Naghavi M, Mensah GA, Ezzati M and Murray CJ. Worldwide epidemiology of atrial fibrillation: a Global Burden of Disease 2010 Study.Circulation . 2014;129:837-47.

2. Chen LY, Chung MK, Allen LA, Ezekowitz M, Furie KL, McCabe P, Noseworthy PA, Perez MV, Turakhia MP, American Heart Association Council on Clinical C, Council on C, Stroke N, Council on Quality of C, Outcomes R and Stroke C. Atrial Fibrillation Burden: Moving Beyond Atrial Fibrillation as a Binary Entity: A Scientific Statement From the American Heart Association. Circulation . 2018;137:e623-e644.

3. Andrade JG, Champagne J, Dubuc M, Deyell MW, Verma A, Macle L, Leong-Sit P, Novak P, BadraVerdu M, Sapp J, Mangat I, Khoo C, Steinberg C, Bennett MT, Tang ASL, Khairy P and Investigators C-DS. Cryoballoon or Radiofrequency Ablation for Atrial Fibrillation Assessed by Continuous Monitoring: A Randomized Clinical Trial. Circulation . 2019.

4. Sanna T, Diener HC, Passman RS, Di Lazzaro V, Bernstein RA, Morillo CA, Rymer MM, Thijs V, Rogers T, Beckers F, Lindborg K, Brachmann J and Investigators CA. Cryptogenic stroke and underlying atrial fibrillation. $N$ Engl J Med . 2014;370:2478-86.

5. Yushan B, Tan BYQ, Ngiam NJ, Chan BPL, Luen TH, Sharma VK, Sia C-H, Dalakoti M, Chong SS, Kojodjojo P and Yeo LLL. Association between Bilateral Infarcts Pattern and Detection of Occult Atrial Fibrillation in Embolic Stroke of Undetermined Source (ESUS) Patients with Insertable Cardiac Monitor (ICM). Journal of Stroke and Cerebrovascular Diseases . 2019;28:2448-2452.

6. Fukuma N, Hasumi E, Fujiu K, Waki K, Toyooka T, Komuro I and Ohe K. Feasibility of a T-ShirtType Wearable Electrocardiography Monitor for Detection of Covert Atrial Fibrillation in Young Healthy Adults.Scientific Reports . 2019;9.

7. Turakhia MP, Desai M, Hedlin H, Rajmane A, Talati N, Ferris T, Desai S, Nag D, Patel M, Kowey P, Rumsfeld JS, Russo AM, Hills MT, Granger CB, Mahaffey KW and Perez MV. Rationale and design of a large-scale, app-based study to identify cardiac arrhythmias using a smartwatch: The Apple Heart Study. Am Heart J . 2019;207:66-75.

8. Bonomi AG, Schipper F, Eerikainen LM, Margarito J, van Dinther R, Muesch G, de Morree HM, Aarts RM, Babaeizadeh S, McManus DD and Dekker LRC. Atrial Fibrillation Detection Using a Novel Cardiac Ambulatory Monitor Based on Photo-Plethysmography at the Wrist. J Am Heart Assoc . 2018;7:e009351.

9. Philippsen TJ, Christensen LS, Hansen MG, Dahl JS and Brandes A. Detection of Subclinical Atrial Fibrillation in High-Risk Patients Using an Insertable Cardiac Monitor. JACC Clin Electrophysiol . 2017;3:15571564 .

10. Ciconte G, Saviano M, Giannelli L, Calovic Z, Baldi M, Ciaccio C, Cuko A, Vitale R, Giacopelli D, Conti M, Lipartiti F, Giordano F, Maresca F, Moscatiello M, Vicedomini G, Santinelli V and Pappone C. Atrial fibrillation detection using a novel three-vector cardiac implantable monitor: the atrial fibrillation detect study.Europace . 2017;19:1101-1108.

11. Nolker G, Mayer J, Boldt LH, Seidl K, V VAND, Massa T, Kollum M, Brachmann J, Deneke T, Hindricks G, Jung W, Brunner KJ, Kraus S, Hummer A and Lewalter T. Performance of an Implantable Cardiac Monitor to Detect Atrial Fibrillation: Results of the DETECT AF Study. J Cardiovasc Electrophysiol . 2016;27:1403-1410.

12. Wasser K, Weber-Kruger M, Jurries F, Liman J, Hamann GF, Kermer P, Uphaus T, Protsenko E, Seegers J, Mende M, Groschel K and Wachter R. The cardiac diagnostic work-up in stroke patients-A subanalysis of the Find-AFRANDOMISED trial. PLoS One . 2019;14:e0216530.

13. Chan PH, Wong CK, Pun L, Wong YF, Wong MM, Chu DW and Siu CW. Head-to-Head Comparison of the AliveCor Heart Monitor and Microlife WatchBP Office AFIB for Atrial Fibrillation Screening in a 
Primary Care Setting. Circulation . 2017;135:110-112.

14. Verma A, Wachter R, Kowey PR, Halperin JL, Gersh BJ, Elkind MSV, Kaplon RE, Ziegler PD, Sherfesee L and Reiffel JA. Changes in Management Following Detection of Previously Unknown Atrial Fibrillation by an Insertable Cardiac Monitor (from the REVEAL AF Study). The American Journal of Cardiology . 2019;124:864-870.

15. Halcox JPJ, Wareham K, Cardew A, Gilmore M, Barry JP, Phillips C and Gravenor MB. Assessment of Remote Heart Rhythm Sampling Using the AliveCor Heart Monitor to Screen for Atrial Fibrillation: The REHEARSE-AF Study. Circulation . 2017;136:1784-1794.

16. Koshy AN, Sajeev JK and Teh AW. Letter by Koshy et al Regarding Article, "Assessment of Remote Heart Rhythm Sampling Using the AliveCor Heart Monitor to Screen for Atrial Fibrillation: The REHEARSE-AF Study". Circulation . 2018;137:2191-2192.

17. Scacciatella P, Jorfida M, Biava LM, Meynet I, Zema D, D'Ascenzo F, Pristipino C, Cerrato P, Giustetto $\mathrm{C}$ and Gaita F. Insertable cardiac monitor detection of silent atrial fibrillation in candidates for percutaneous patent foramen ovale closure. J Cardiovasc Med (Hagerstown) . 2019;20:290-296.

18. Iwata T, Todo K, Yamagami H, Morimoto M, Hashimoto T, Doijiri R and Furuya H. High Detection Rate of Atrial Fibrillation With Insertable Cardiac Monitor Implantation in Patients With Cryptogenic Stroke Diagnosed by Magnetic Resonance Imaging. J Stroke Cerebrovasc Dis . 2019;28:2569-2573.

19. Goldenthal IL, Sciacca RR, Riga T, Bakken S, Baumeister M, Biviano AB, Dizon JM, Wang D, Wang KC, Whang W, Hickey KT and Garan H. Recurrent atrial fibrillation/flutter detection after ablation or cardioversion using the AliveCor KardiaMobile device: iHEART results. J Cardiovasc Electrophysiol . 2019.

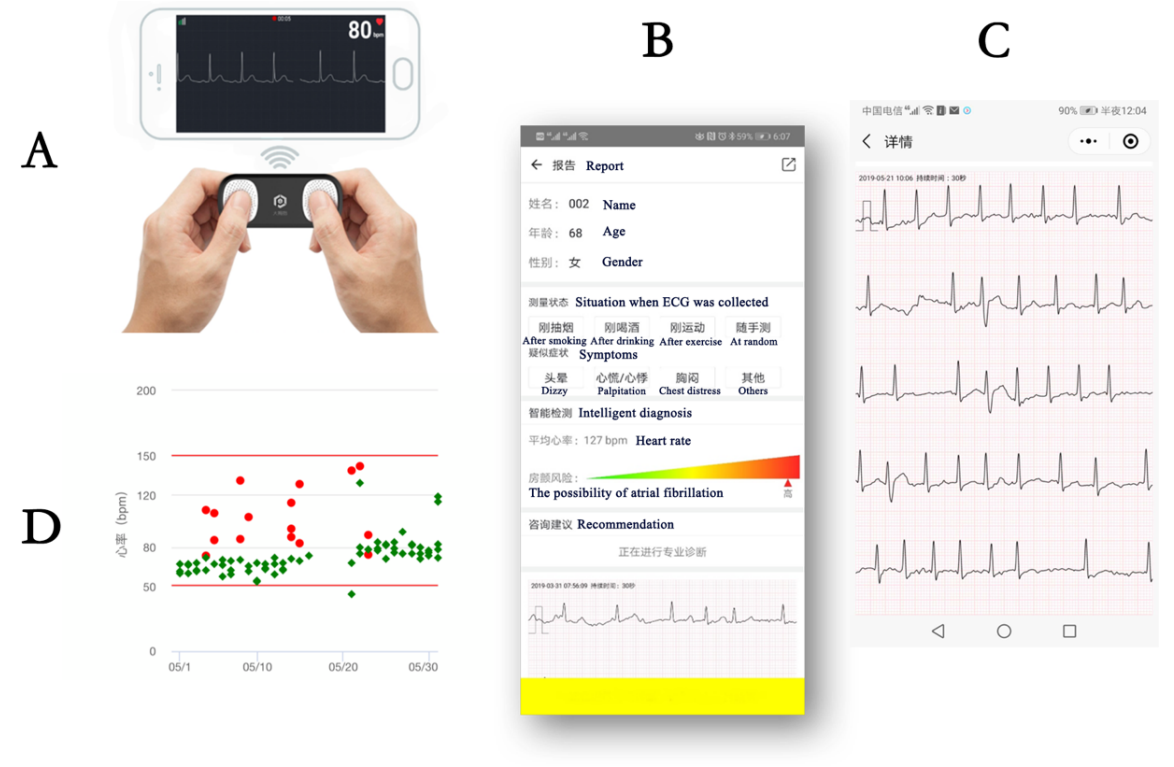

Figure 1 A: The BigThumb system. With two thumbs of users on the device, a bECG will be collected. B: Software interface of the BigThumb. Users are asked if there is a symptom when bECG is collected. C: A bECG collected by a patient with atrial fibrillation. D: Summary of bECG in one-month duration. The red points show the bECG with atrial fibrillation and the green points show the sinus rhythm.

Table 1. Characteristics Between Patients in BT and TF Groups 


\begin{tabular}{llll}
\hline Variables & BT Group $(\mathrm{N}=109)$ & TF Group(N=109) & P-Value \\
\hline Male (\%) & $84(77.1 \%)$ & $70(64.2 \%)$ & 0.296 \\
Age & $62.3 \pm 8.5$ & $64.1 \pm 10.3$ & 0.165 \\
Paroxysmal AF (\%) & $65(59.6 \%)$ & $76(69.7 \%)$ & 0.119 \\
BMI $\left(\mathrm{Kg} / \mathrm{m}^{2}\right)$ & $24.9 \pm 2.7$ & $24.5 \pm 2.8$ & 0.347 \\
$\mathrm{CHA}_{2} \mathrm{DS}_{2}$-VASc & $1.7 \pm 1.5$ & $2.0 \pm 1.4$ & 0.192 \\
$\mathrm{HAS}_{\mathrm{BLED}}$ & $1.2 \pm 1.0$ & $1.3 \pm 1.0$ & 0.889 \\
Scr (umol/L) & $77.4 \pm 15.3$ & $79.7 \pm 22.3$ & 0.375 \\
GFR (ml/min) & $88.4 \pm 15.3$ & $84.3 \pm 21.4$ & 0.103 \\
ALT (U/L) & $27.3 \pm 17.9$ & $27.8 \pm 26.0$ & 0.878 \\
AST (U/L) & $21.1 \pm 10.6$ & $21.8 \pm 12.2$ & 0.677 \\
LAD (cm) & $4.1 \pm 2.6$ & $3.9 \pm 0.5$ & 0.443 \\
EF (\%) & $61.2 \pm 5.0$ & $60.4 \pm 8.0$ & 0.397 \\
Follow-Up Duration (days) & $344.0 \pm 60.5$ & $346.8 \pm 59.9$ & 0.737 \\
Procedure Duration (min) & $155.6 \pm 46.1$ & $157.8 \pm 67.4$ & 0.776 \\
Fluoroscopy Time (min) & $10.5 \pm 5.9$ & $9.9 \pm 6.0$ & 0.460 \\
Radiation Dose (mGy) & $245.7 \pm 173.9$ & $229.9 \pm 178.5$ & 0.511 \\
\hline
\end{tabular}

$\mathrm{BMI}=$ body mass index; Scr $=$ serum creatinine GFR $=$ glomerular filtration rate; ALT $=$ alanine transaminase; $\mathrm{AST}=$ aspartate transaminase; $\mathrm{LAD}=$ left atrial diameter; $\mathrm{EF}=$ ejection fraction. Continuous variables were compared using the two-tailed Student's t-test, and categorical variables were compared using the $\chi^{2}$ test or Fischer's exact test.

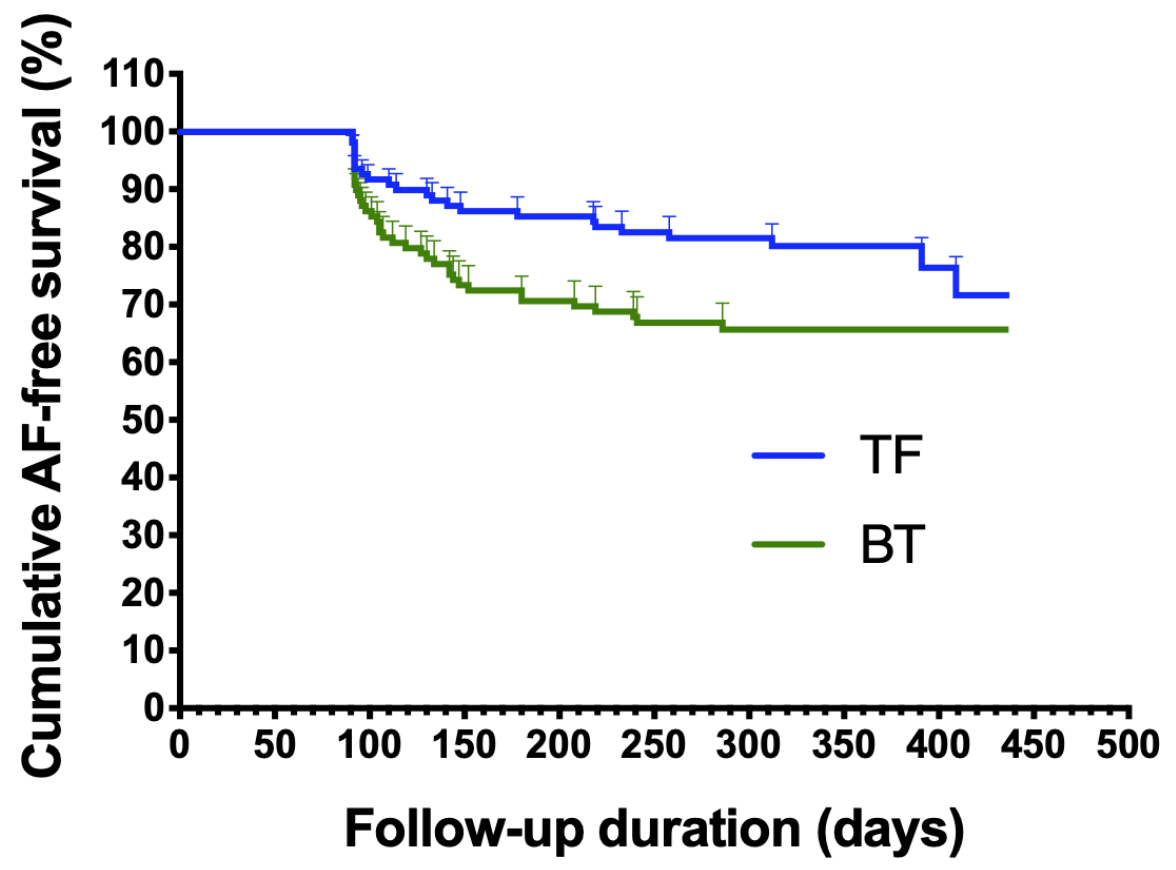

Figure 2 The Kaplan-Meier survival curve for the primary endpoint

Kaplan-Meier survival curve and log rank analysis were performed. $\mathrm{P}=0.022$.

Table 2. Cox Regression Analysis with AF Recurrence 


\begin{tabular}{|c|c|c|c|c|c|}
\hline Variable & $\begin{array}{l}\text { Univariable } \\
\text { Analysis }\end{array}$ & $\begin{array}{l}\text { Univariable } \\
\text { Analysis }\end{array}$ & & $\begin{array}{l}\text { Multivariable } \\
\text { Analysis }\end{array}$ & $\begin{array}{l}\text { Multivariable } \\
\text { Analysis }\end{array}$ \\
\hline & $\begin{array}{l}\beta \text { Coefficient } \\
(95 \% \text { CI })\end{array}$ & $\mathrm{P}$ & & $\begin{array}{l}\beta \text { Coefficient } \\
(95 \% \mathrm{CI})\end{array}$ & $\mathrm{P}$ \\
\hline Follow-up & 0.33 (0.15 to & 0.006 & Follow-up & 0.41 ( 0.18 to & 0.029 \\
\hline Strategy & $0.73)$ & & Strategy & $0.91)$ & \\
\hline $\mathrm{LAD}$ & $\begin{array}{l}2.79 \text { (1.60 to } \\
4.89)\end{array}$ & 0.001 & $\mathrm{LAD}$ & $\begin{array}{l}2.34(1.20 \text { to } \\
4.57)\end{array}$ & 0.013 \\
\hline
\end{tabular}

$\mathrm{LAD}=$ left atrial diameter

For Cox regression, univariable regression was performed first. Variables with a P-value of $<0.2$ were included in the multivariable model (Follow-up strategy and LAD). A level of significance was set at $<0.05$ for all reported P-values, and confidence intervals were calculated at the $95 \%$ level.

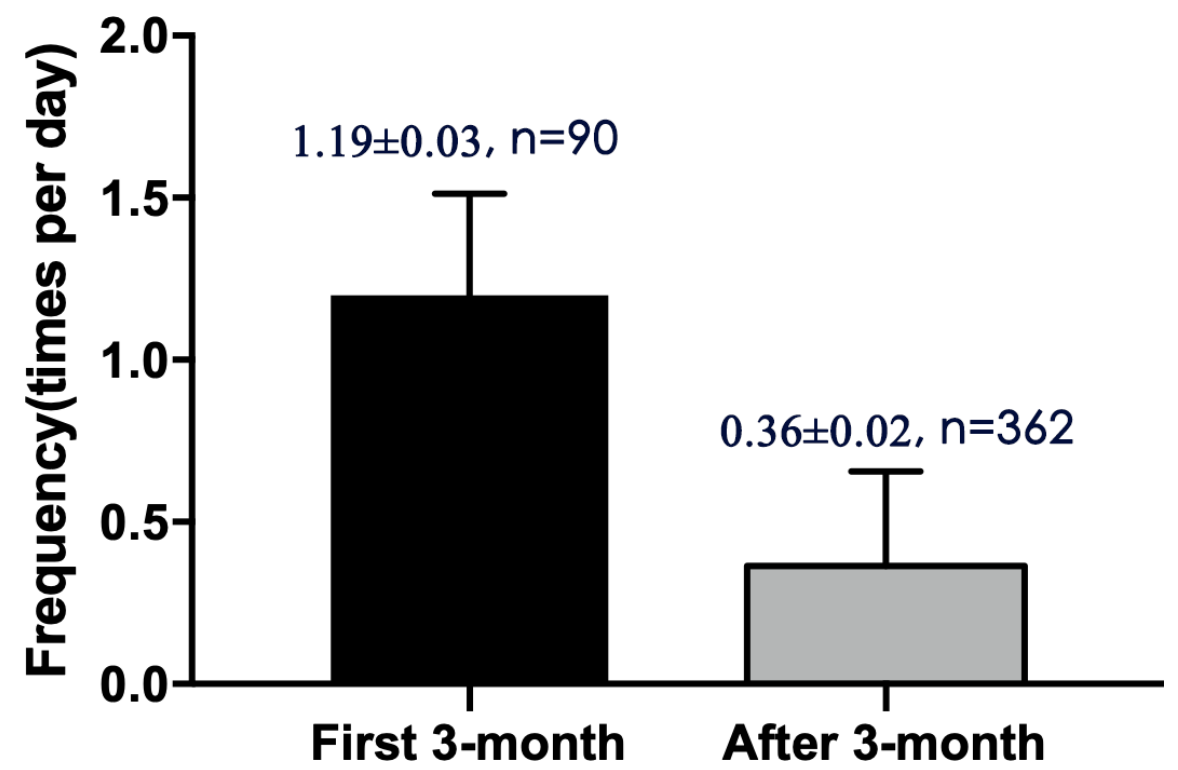

Figure 3 The monitor frequency in different duration of follow-up

Frequency is expressed as mean \pm standard deviation, and compared using the two-tailed Student's t-test, $\mathrm{P}<0.0001$ 


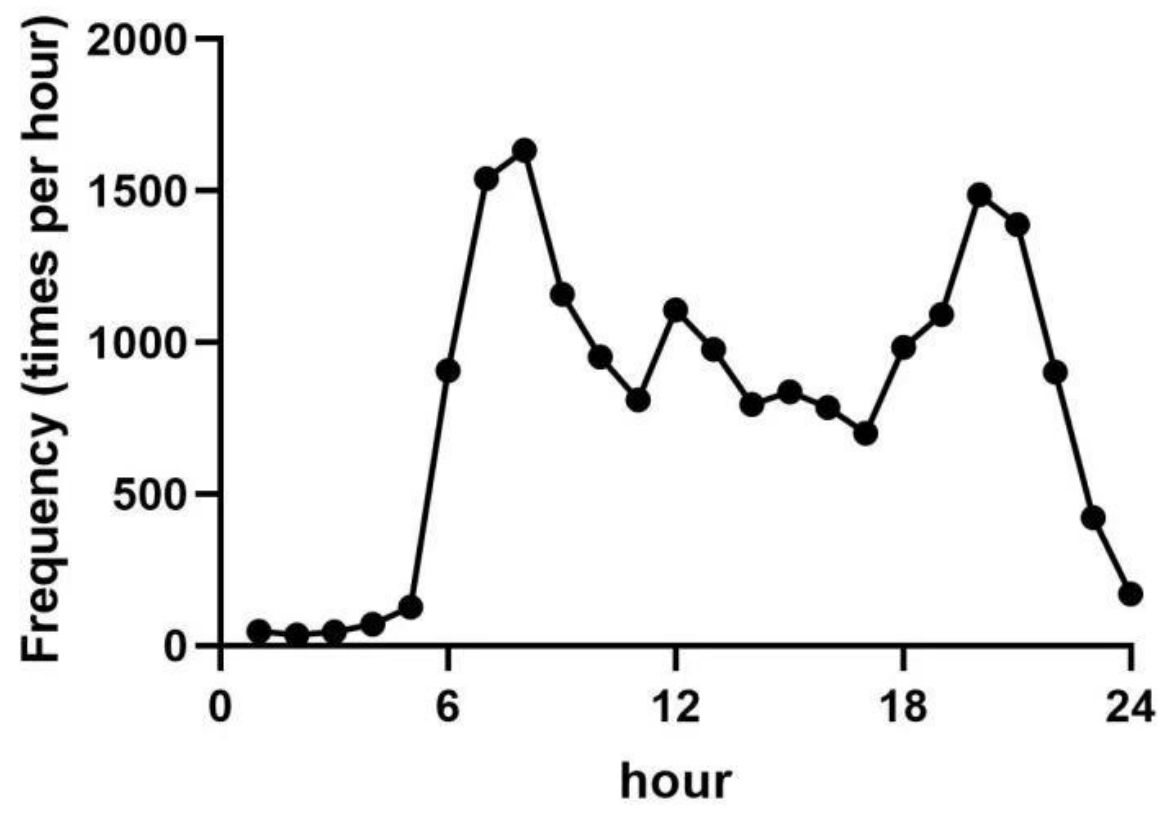

Figure 4 The monitor frequency collected in 24-hour duration 